\title{
ECoG Correlation Variation for Epilepsy Research
}

Ziv Yekutieli ${ }^{1,2^{*}}$ and Eshel Ben-Jacob ${ }^{1}$

${ }^{1}$ School of Physics and Astronomy, Faculty of Exact Sciences, Tel Aviv University, Tel Aviv, Israel

${ }^{2}$ School of Electrical Engineering, Faculty of Engineering, Tel Aviv University, Tel Aviv, 6997801, Israel

"Corresponding author: Yekutieli Z, School of Electrical Engineering, Faculty of Engineering, Tel Aviv University, Tel Aviv, 6997801, Israel, Tel: +972 4 8580038; E-mail: ziv.yekutieli@gmail.com

Received date: January 05, 2018; Accepted date: January 11, 2018; Published date: January 18, 2018

Copyright: @ 2018 Yekutieli Z, et al. This is an open-access article distributed under the terms of the Creative Commons Attribution License, which permits unrestricted use, distribution, and reproduction in any medium, provided the original author and source are credited.

\begin{abstract}
Epilepsy is a well-known affliction characterized by recurrent, unprovoked seizures affecting 1-2\% of the population. Other than the actual seizure and the risks involved in it, the sudden and unpredictable nature of the seizure is one of the most disabling aspects of epilepsy. As such, finding a method capable of predicting epileptic seizures would open new therapeutic possibilities.

When using electrocorticography for capturing brain activity of epileptic patients, usually, it is the clear increase in the brain activity that is recorded during the seizure. The data is usually used in order to find the probable focus of the seizure. Using the same data, we have applied a method that was used for another disorder (Ataxia Telangiectasia), for obtaining quantitative information about changes in the network correlation, rather than network activity. We show that this provides insight for the epileptic brain behavior, demonstrating that other locations of the brain are involved in the seizure other than the focus, and that there might be early indications for the seizure. These findings can potentially be used in order to decrease seizure likelihood.
\end{abstract}

Keywords: Epilepsy; Electrocorticography; Neural network; Connectivity; Correlation

\section{Abbreviations:}

- EEG: Electroencephalography; ECoG: Electrocorticography.

\section{Introduction}

Epilepsy is a well-known affliction characterized by recurrent, unprovoked seizures affecting $1-2 \%$ of the population [1,2]. Epilepsy is not a single disorder, but rather a group of neurological disorders characterized by epileptic seizures, that can range from nearly undetectable events to long periods of vigorous shaking [3].

The cause of most epilepsy cases is unknown [4]. Few cases are genetically related, and some people develop epilepsy as a result of brain injury, stroke, brain tumor, drug abuse and even autoimmune diseases [5-7]. Epilepsy is often confirmed by electroencephalography (EEG), but a normal test is not enough to rule out the disease [8-10].

When medication is insufficient to control the seizures, surgery remains the only alternative. In order to identify the foci of the seizure electrocorticography (ECoG) is needed [11]. Due to the risk and complexity involved in ECoG, it is strictly used for clinical purposes. As a result, this has been the main focus of ECoG studies as well, where most of the attention is given to spatial identification of the foci [12]. Therefore, there is no ECoG data from healthy individuals. In this work, we are using ECoG data in order to examine and quantify other properties of the network, other than the seizure foci, and due to the fact that there is no control group, we compare the data recording between the seizures, with the date recorded during the seizures.

Other than the actual seizure and the risks involved in it, the sudden and unpredictable nature of the seizure is one of the most disabling aspects of epilepsy. Thus, finding a method capable of predicting epileptic seizures would open new therapeutic possibilities, and this can be attempted by analyzing the neural network stability [13-17].

\section{Experimental Procedures}

\section{Patients}

Patients with drug-resistant epilepsy may be candidates for resective surgery of an epileptic focus to minimize the frequency of seizures. Prior to resection, the patients undergo monitoring using ECoG subdural electrodes for two purposes: first, to localize the epileptic focus, and second, to identify nearby critical brain areas which should not be removed due to high risk of long-term functional deficits. The patient is typically recorded over a period of 5-12 days, the data is used for localizing the epileptic foci. Electrical stimulation via the implanted electrodes allows clinicians to map eloquent cortex. In some occasions, means are taken to evoke a seizure which is essential for the localization procedure to succeed. At the end of the monitoring period, the electrodes are removed and therapeutic resection is performed [17].

We have analyzed data of four epilepsy patients who were not sufficiently responsive to medicine and hence required surgical intervention. Three of these patients were females and one was a male, all young, between the age of 10 and 20 years old. The data was provided to us by Prof. Oren Sagher, a neurosurgeon at the University of Michigan hospital. Established standards for the ethical treatment of human research subjects were approved by the institutional review board at the University of Michigan hospitals. 


\section{Epileptogenic zone}

Localization of the epileptogenic zone, defined as the volume of brain tissue necessary and sufficient for the generation of seizures, has traditionally depended on scalp EEG recordings which often do not provide sufficient accuracy and resolution. In these cases, further evaluations rely on invasive brain recordings, using arrays of ECoG electrodes placed directly on the surface of the brain, and/or recordings from depth electrodes inserted deeper into the brain to regions such as the hippocampus or the medial temporal lobe [18]. The electrodes are spatially distributed over the suspected focal regions (as determined from conventional scalp EEG recordings and other imaging techniques), and are kept in place for a long period of time so that the focus or foci could be localized. The common clinical procedure for localization and assessment of epileptogenic foci is a visual inspection and interpretation of the intracranially recorded signals by experienced clinicians with several advanced analysis methods developed over the years [15,19-21].

\section{Data acquisition}

The ECoG systems used record up to 128 channels. The channels were divided into $4 \times 8$ or $8 \times 8$ arrays, and strips of up to 8 electrodes. The arrays and strips were placed based upon previous examination of the patients' brain by PET and MRI. The signals were initially recorded at $400 \mathrm{~Hz}$, digitized to $112 \mathrm{~Hz}$ and low passed filtered at $40 \mathrm{~Hz}$. Seizure data included at least two episodes per patient, and data between seizures ranged from 30 to $160 \mathrm{~min}$ per patient. Typically, all ECoG channels are referenced to a common reference electrode.

Once the data is obtained, it is visually screened for artifacts. With the use of spectral analysis (univariate linear measures) it has been shown that distinct activity at specific frequency bands is relevant for epilepsy diagnosis [22-26].

\section{Results}

\section{Raw ECoG data}

We start by presenting the analog output of the ECoG signal, making sure that the seizures are properly tagged in the data. Higher amplitudes and frequencies are characteristics of a seizure [27,28], as can be seen in the example in (Figure 1).

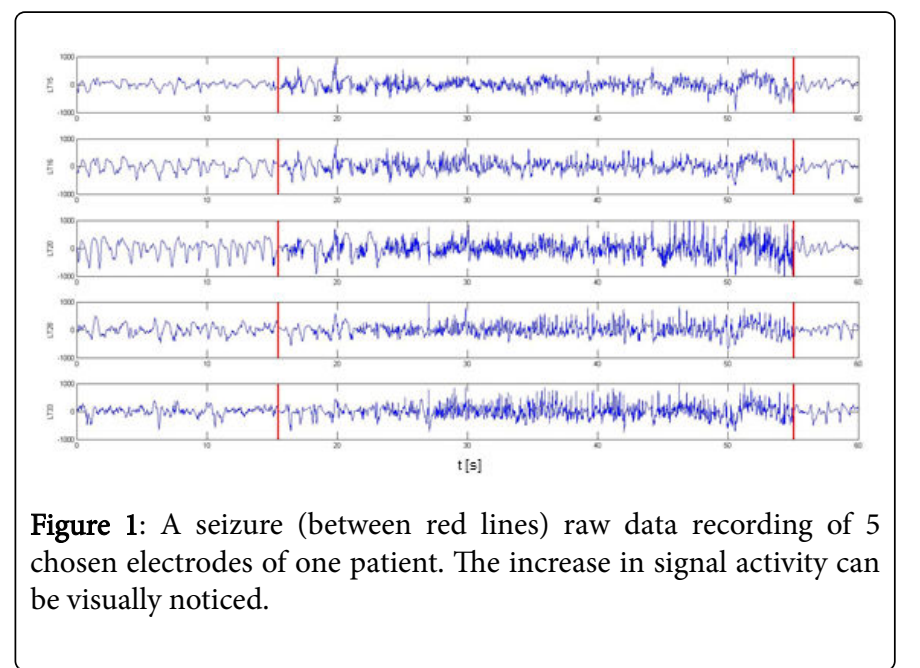

\section{ECoG energy}

The magnitude of the analog signal in ECoG is determined by the neuronal activity: the more spikes per seconds we have in the electrode receptive filed, the larger the signal is. We therefore start by analyzing the energy of the signals at hand. Figure 2 shows the ECoG signal energy for four patients, one seizure for each patient.

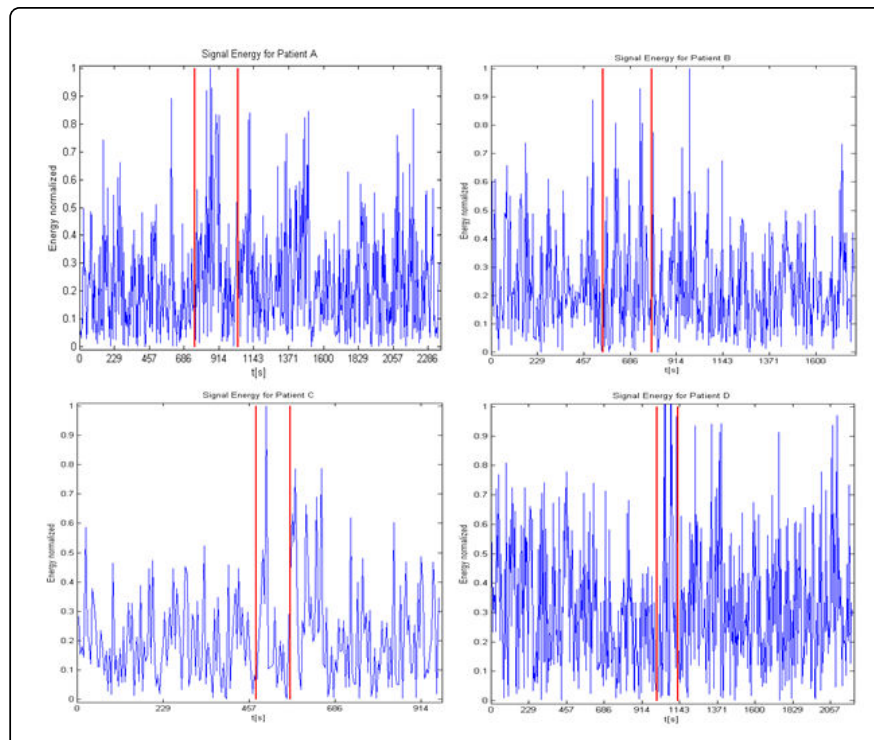

Figure 2: ECoG overall energy of all the electrodes for each patient, one seizure per patient. The signals are normalized for each patient separately as we care about the relative change in energy. The seizure is marked between two red lines.

For these patients, we measure an increase of about $80-120 \%$ in the average energy during the seizure relative to between seizures. This means that during the seizure, the neuronal activity is about double of the norm in these cases. Other research indicates that the signal can increase by even more, offering various methods to use these signals for seizure detection $[14,29]$.

\section{Network connectivity}

It is virtually impossible to map the neuronal network of the human brain in a mechanical sense, namely, mark each connection between each neuron and the strength of each connection. When using multielectrode arrays, one can map the neural network connectivity by analyzing the electrical activity of the nodes in that network, where clusters identified by phase synchronization analysis are mapped into real clusters of neurons [30-32]. This is also the case for ECoG signals, and we can use brain network activity to reveal the network topology $[33,34]$. Network connectivity is therefore used for foci localization algorithms, in a manner that does not only look at the location with the highest energy, but the source of the seizure in the network formation $[15,18,34]$. It is therefore reasonable to see if and how the network changes during seizures and between them.

We begin by mapping the network in continuous timeframes. First, each channel was normalized in order to avoid artifacts and DC shifts. Then, we calculated the Pearson correlation between all electrode combinations: 


$$
r_{x y}=\frac{1}{n-1} \sum \frac{\left(x_{i}-\bar{X}\right)\left(y_{i}-\bar{Y}\right)}{s_{x} s_{y}}
$$

Where $\mathrm{n}$ is the total number of samples, $\mathrm{x}_{\mathrm{i}}\left(\mathrm{x}_{1}, \mathrm{x}_{2}, \ldots ., \mathrm{x}_{\mathrm{n}}\right)$ are the amplitude values of channel $x$ and $y_{i}$ are the amplitude values of channel $y . S_{x}, S_{y}$ are the corresponding standard deviations of each electrode. The correlation was calculated with a sliding window of time with an overlap of $\mathrm{n} / 2$ samples. We have examined timeframes between half a second and 32 seconds and found that 4 seconds provide sufficient resolution while including enough data to eliminate noise. Other methods for measuring the network formation were also examined such as phase synchronization measurement [35], they produced similar results while being more computationally exhaustive.

\section{Epilepsy network variation}

Having created a correlation snapshot for each network, we examine the variation in the network in each of the patients, using a similar method to Phase Synchronization Variation [31]. In short, we first generate a dendrogram from the normal network correlation matrix, which serves as the network baseline. This dendrogram marks the order of the links between the different areas of the brain, according to the overall and normal brain behavior. Then, we generate correlation matrices for 4 seconds timeframes for the entire data, during the seizures and between them. We generate dendrograms for all those windows, according to the same order as was obtained from the baseline matrix. We normalize all the dendrograms (for each patient individually), so that we will get a standard measure of change between 0 (no change in correlation) and 1 (the maximum change in correlation for that patient). Finally, we calculate the delta between each pair of matrices (the baseline and the current window) as can be seen in Figure 3.

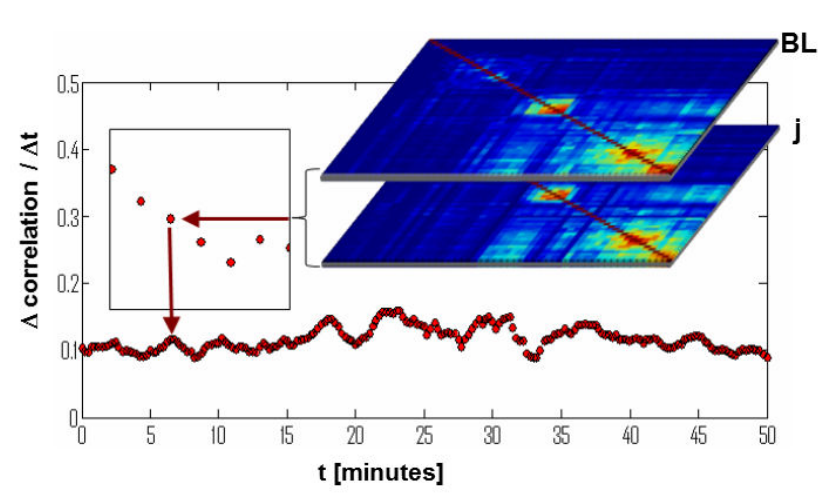

Figure 3: Correlation Variation metric: Each correlation matrix, $j$, representing the correlation state of the network in a 4 seconds window, is subtracted from the baseline matrix BL. Each "pixel" which represents the link between two nodes, is thus compared to the same pixel in the overall network, and we measure the total absolute change.

Figure 4 shows the network correlation variation over one session for each patient. The epileptic seizure occurs between the two red lines as reported by the clinicians and validated through the activity analysis.

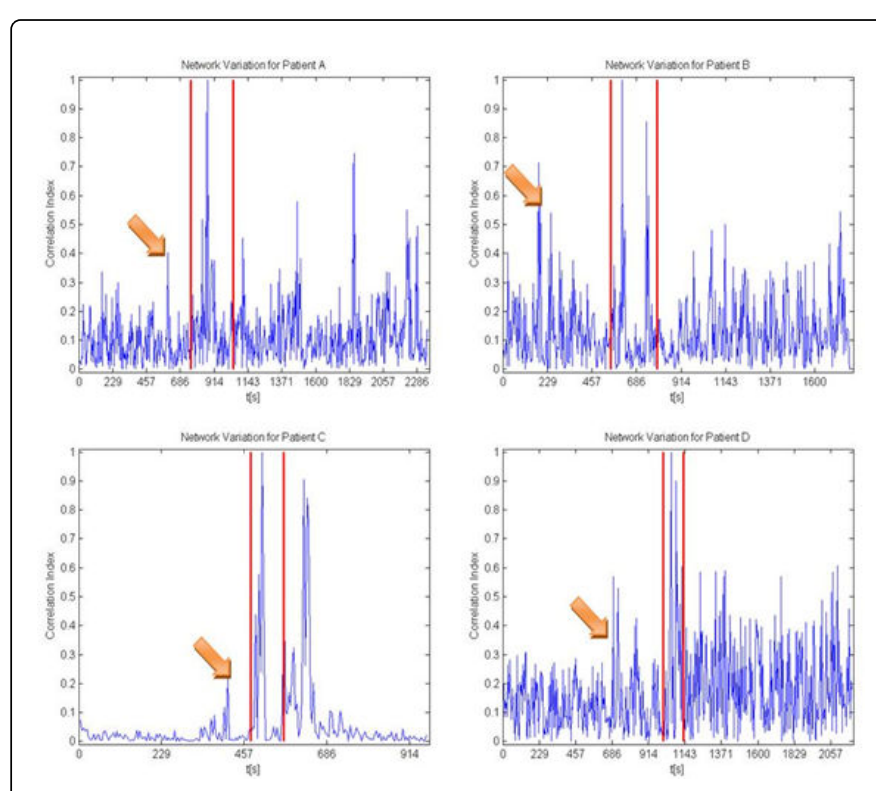

Figure 4: Network correlation variation of four epilepsy patients. Each window represents a typical seizure that was recorded during the ECoG session. The seizure Start and End times are marked by the red lines. The duration of the seizure was provided by the neurologists monitoring the patients. The orange arrows indicate the pre-seizure signal we will refer to below.

Monitoring the patient's networks through the correlation analysis teaches us that the correlation variation between seizures is relatively low; correlation variation during a seizure is very high. In other words, during normal brain activity, each region of the brain works while keeping the same correlation with the other regions, it can be high or low, but it varies relatively little. During the epileptic seizure, these correlations are changing more rapidly as we experience bursts of highly synchronized activity. We see that the correlation variation signal is much more prominent than activity: While the average activity during seizures was larger by $80-120 \%$ than the average activity between seizures, the average correlation variation during seizures is larger by about $160 \%-230 \%$ from the correlation variation between seizures.

\section{Epilepsy network "pre-seizure" variation}

The seizures durations are labeled by the clinician, namely, while observing the patient and recording the data, the clinician will mark when each seizure begins and ends, so we can track it on our analysis as well (the red lines marked in the figures). Once the seizure occurs, a window of data including the time of the seizure itself, with the addition of sometime before and after the seizure, is used for the analysis described above. Interestingly, if we examine the network correlation variation throughout the recording session, prior to the seizure itself we notice an increase in the network correlation variation which is marked by the orange arrows in Figure 4, we refer to it as the pre-seizure signal. This phenomenon is not explained at this time. We also notice high network correlation variation after the seizure itself is reported to stop, as an "after shock effect" of the seizure until it decays completely and the patient recovers. These pre-seizure signs might be meaningful not only in order to better explain why the seizure takes 
place, but can also provide an important tool for seizure prevention technologies as they precede the seizure.

\section{Epilepsy pre-seizure partial network variation}

During the pre-seizure hyper-correlation-variation events, we notice areas in the network which present large correlation variations which are not in the seizure focus. While the focus is typically displaying high correlation throughout the recording, these off-diagonal "hotspots" have typically low correlation between seizures and form high correlation just prior to the seizure and during it (Figure 5).

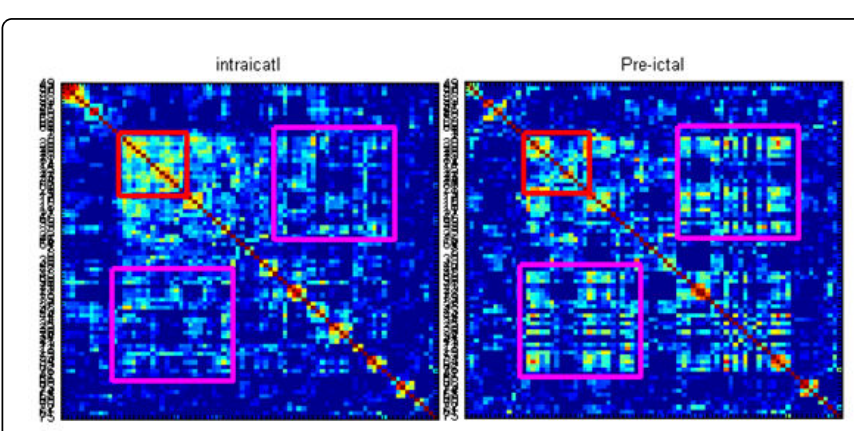

Figure 5: ECoG presenting off-diagonal "hotspots": On the left, an example of a correlation dendrogram presented between seizures. The red rectangle marks the foci as determined by the physician. On the right, another correlation dendrogram (ordered as before), taken during one of the early hyper-correlation peaks (pre-seizure). The off-diagonal purple rectangles mark an area that was previously showing low correlation and now, a short time before the seizure, shows several clusters of higher correlation.

We suggest that these areas in the network should get as much attention as the foci get: They might not be the areas that evoke the seizure, but they seem to allow it to propagate.

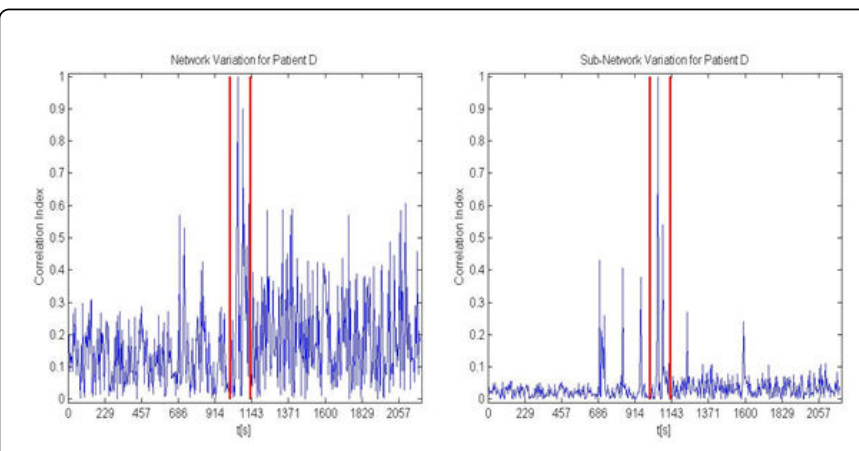

Figure 6: ECoG Sub-Network correlation: On the left, the same chart as Figure 4 for patient D, we measure the network correlation variation all channels available (96 in this example). On the right, we monitor the network variation by using only the 4 channels with the highest correlation variation, presenting a much clearer preseizure signal.

This analysis is also useful for potential treatment: As the offdiagonal hotspots present high correlation variation, we have looked into reducing the number of channels needed in order to detect the network correlation variation. Choosing the four channels that demonstrated the highest correlation variation (from the off-diagonal hotspots) we were still able to detect the seizure, while getting even better SNR (Figure 6).

This means that we can obtain early indication for a potential seizure by monitoring few electrodes. While for some patients and seizures even the correlation of 2 electrodes was sufficient to provide the same signal, for all patients and seizures that we have examined, using less than 4 electrodes will not detect all the seizures with sufficient margin relative to normal correlation levels.

\section{Supporting Evidence}

Support to our observations is offered by similar results reported by Burns et al. [36]. While still focusing on the foci localization, Burns claims that "a subset of nodes is isolated from the network at seizure onset and becomes more connected with the network toward seizure termination". These subsets of nodes are what we refer to as the offdiagonal "hotspots". If these clusters were fixed ones, they would have appeared on the dendrogram diagonal as the dendrogram is ordered according to the overall correlation (the correlation of the entire normal activity). Burns also suggests that these connectivity changes are behaving in repetitive patterns, making them useful for early detection and making seizure prevention more likely.

As for the pre-seizure signals we notice, here we might suggest evidence from a sensation that the patients themselves experience, usually referred to as "aura". Aura is a term that is used to describe a subjective premonition that patients sense some time before the onset of the seizure. The sensation is a rather elusive one and it varies between one patient to another, it can be manifested by a range of sensations starting with a mild headache and spanning to some odd signals picked by our senses, such as phantom sounds [37]. The amount of time before the seizure that aura occurs also varies between one patient to another.

As this sensation is difficult to quantify and measure, the exact probability of aura is undetermined, but it is not a rare phenomenon: recent research indicates that it happens for at least $21 \%$ of the patients [38] and up to $67 \%$ of the patients [39]. We should stress that we do not have any reports from our patients about aura experience in correlation to our data, as they were not asked about it. We suggest that pre-seizure network correlation variation and off-diagonal "hotspots" are related to neuronal manifestation of the aura sensation and potentially are the neurological evidence for this subjective phenomenon. This could be examined in future research.

\section{Discussion}

We have used ECoG data from four epilepsy patients in order to examine how the neural network fluctuates during a seizure and between seizures. As the network correlation represents network topology, we have used the correlation and presented a metric for measuring the network changes.

Epileptic seizures are indeed manifested by an increase in the activity of the network, as indicated by the energy of the signals. However, even more noticeably, it is manifested by a larger increase in the neural network correlation variation, so we can refer to the seizure more as a hyper-correlation-variation event than a hyper-activity event. Also, while the foci of the seizure might trigger it, it takes the entire network to take part in allowing the seizure to propagate, with some areas having a dominant role in the seizure propagation. This 
Page 5 of 6

decrease in the brain neuronal stability (increase in correlation variation) is very likely the cause of epilepsy most prominent symptom - the seizure. As large portions of the brain start and stop acting simultaneously, most of the muscles are contracted and relaxed alternatively, and the patient is unable to control his or her body. The correlation "wave" is rapidly circulating around the brain with one region activating another until the neurons have exhausted their neurotransmitters and cannot keep on re-activating each other. Activity and correlation might seem related, but they are not, and this is key to understanding epilepsy and other neurological disorders. An analogy for increase in activity might be provided by a group of people who stand still and then start running, each on his own speed and direction. In that aspect, correlation would be that the same people will start marching together. Even if they do so very slowly, their correlation is high. If they start falling in and out of the same marching rhythm, that's when their correlation variation increases (stability decreases).

The brain has to maintain a balance between stability and flexibility in its network correlation. For some reason, in the brain of epileptic patients, there are events of hyper correlation variation. Whether this is due to a higher correlation baseline, or due to over sensitivity for external stimulus, is a subject for further research.

\section{Conclusions}

While being a small sample of patients, this work has been done to examine the application of measuring network variation for epilepsy. Doing so, we have noticed that this representation of the ECoG signals can indeed reveal important aspects of the brain activity in both space and time. Correlation variation provides a stronger signal than activity for differing between normal activity and seizures. We have noticed a change in correlation variation occurring some time before the epileptic seizure takes place, during the "pre-seizure" period, but it is not clear if this will be valid for all epilepsy patients. The aura sensation which is experienced by many patients might suggest that the preseizure period is common. We have demonstrated that it is sufficient to measure the correlation of few channels in order to depict the correlation variation early signals. These observations suggest that future treatment of epilepsy which does not rely upon cortical tissue removal but rather upon stimulation [40-42] can depict the epileptic seizure with very few electrodes by monitoring their correlation. Doing so, we might be able to predict the seizure and eliminate it before it starts. The off-diagonal "hotspots", the high correlation-variation areas in the brain, are also relevant for potential treatment: in cases that the seizure foci cannot be removed, because it is located in a critical brain area, removing the off-diagonal "hotspots" might allow preventing the seizure from advancing. If stimulation is to be used, stimulating these areas when a seizure is detected (or predicted) will cause them to be exhausted when the seizure wave reaches them, causing the wave to decay.

The authors would like to thank Prof. O. Sagher from the University of Michigan, for sharing his ECoG recordings with us.

This research did not receive any specific grant from funding agencies in the public, commercial, or not-for-profit sectors.

\section{References}

1. Holmes MD, Browne TR, Gregory L (2008) Handbook of epilepsy (4th ed.). Philadelphia: Lippincott Williams \& Wilkins. P: 7.
2. Hawley SR, Ablah E, Hesdorffer D, Pellock JM, Lindeman DP (2015) Prevalence of pediatric epilepsy in low-income rural Midwestern counties. Epilepsy Behav 53: 190-196.

3. Chang BS, Lowenstein DH (2003) "Classification of neural action potentials network," Computation in Neural Systems.

4. Timofeev I, Steriade M (2004) Neocortical seizures: initiation, development and cessation. Neuroscience 123: 299-336.

5. Irani SR, Buckley C, Vincent A, Cockerell OC, Rudge P, et al. (2008) Immunotherapy-responsive seizure-like episodes with potassium channel antibodies. Neurology: 71.

6. Quek AM, Britton JW, McKeon A, So E, Lennon VA, et al. (2012) Autoimmune epilepsy: clinical characteristics and response to immunotherapy. Arch Neurol 69: 582-593.

7. Greco A, Rizzo MI, De Virgilio A, Conte M, Gallo A, et al. (2016) Autoimmune epilepsy. Autoimmun Rev 15: 221-225.

8. Holmes MD, Tucker DM, Quiring JM, Hakimian S, Miller JW, et al. (2010) Comparing noninvasive dense array and intracranial electroencephalography for localization of seizures. Neurosurgery 66 : 354-362.

9. Longo, Dan L (2012) Harrison's principles of internal medicine. McGrawHill.

10. Caballero-Gaudes C, Van de Ville D, Grouiller F, Thornton R, Lemieux L, et al. (2013) Mapping intraictal epileptic discharges using mutual information between concurrent EEG and fMRI. Neuroimage 68: 248-262.

11. Sirvern JI (2014) Electrocorticogram (ECOG). Encyclopedia of the Neurological Sciences.

12. Lai Y, van Drongelen W, Hecox K, Frim D, Kohrman M, et al. (2007) Cortical activation mapping of epileptiform activity derived from interictal ECoG spikes. Epilepsia 48: 305-314.

13. Elger CE (2001) Future trends in epileptology. Current Opini Neurol 14: 185-186.

14. Le Van Quyen M, Martinerie J, Navarro V, Boon P, D'Havé M, et al. (2001) Anticipation of epileptic seizures from standard EEG recordings. Lancet 357: 183-188.

15. Mormann F, Andrzejak RG, Elger CE, Lehnertz K (2007) Seizure prediction: the long and winding road. Brain 130: 314-333.

16. Osorio I, Frei MG (2008) Realtime Detection, Quantification, Warning, and Control of Epileptic Seizures. US Neurol 4: 67-70.

17. Hill NJ, Gupta D, Brunner P, Gunduz A, Adamo MA, et al. (2012) Recording Human Electrocorticographic (ECoG) Signals for Neuroscientific Research and Real-time Functional Cortical Mapping. J Vis Exp 26.

18. Engel AK, Moll CKE, Fried I, Ojemann GA (2005) Invasive recordings from human brain: Clinical insights and beyond. Nat Rev Neurosci 6: 35-47.

19. Quian Quiroga R, Kraskov A, Kreuz T, Grassberger P (2002) Performance of different synchronization measures in real data: A case study on electroencephalographic signals. Phys Rev E Stat Nonlin Soft Matter Phys 65.

20. Towlea VL, Syeda I, Bergera C, Grzesczcukb R, Miltona J, et al. (1998) Identification of the sensory/motor area and pathologic regions using ECoG coherence. Electroencephalography and Clinical Neurophysiology 106: 30-39.

21. Towle VL, Carder RK, Khorasani L, Lindberg D (1999) Electrocorticographic coherence patterns. J Clin Neurophysiol 16: 528-547.

22. Panet-Raymond D, Gotman J (1990) Asymmetry in delta activity in patients with focal epilepsy. Electroencephalogr Clin Neurophysiol 75: 474-481.

23. Allen PJ, Fish DR, Smith SJ (1992) Very high-frequency rhythmic activity during SEEG suppression in frontal lobe epilepsy. Electroencephalogr Clin Neurophysiol 82: 155-159. 
24. Gambardella A, Gotman J, Cendes F, Andermann F (1995) Focal intermittent delta activity in patients with mesiotemporal atrophy. Epilepsia 36: 122-129.

25. Worrell GA, Parish L, Cranstoun SD, Jonas R, Baltuch G, et al. (2004) High-frequency oscillations and seizure generation in neocortical epilepsy. Brain 127: 1496-1506.

26. Towle VL, Hunter JD, Edgar JC, Chkhenkeli SA, Castelle MC, et al. (2007) Frequency domain analysis of human subdural recordings. J Clin Neurophysiol 24: 205-213.

27. Ravish DK, Devi SS, Krishnamoorthy SG, Karthikeyan MR (2013) Detection of Epileptic Seizure in EEG Recordings by Spectral Method and Statistical Analysis. J Applied Scienc 13: 207-219.

28. Gajic D, Djurovic Z, Gennaro SD, Gustafsson F (2014) Classification of EEG signals for detection of epileptic seizures based on wavelets and statistical pattern recognition. Linköping University Post Print.

29. McSharry PE, He T, Smith LA, Tarassenko L (2002) Linear and nonlinear methods for automatic seizure detection in scalp electroencephalogram recordings. Med Biol Eng Comput 40: 447-461.

30. Baruchi I, Volman V, Raichman N, Shein M, Ben-Jacob E (2008) The emergence and properties of mutual synchronization in in-vitro coupled cortical networks. Eur J Neurosci 28: 1825-1835.

31. Levine-Small N, Yekutieli Z, Aljadeff J, Boccaletti S, Ben-Jacob E (2011) Reduced synchronization persistence in neural networks derived from atm-deficient mice. Front Neurosci 5: 46.

32. Segev R, Baruchi I, Hulata E, Ben-Jacob E (2004) Hidden Neuronal Correlations in Cultured Networks. Phys Rev Lett 92.
33. Centeno M, Carmichael DW (2014) Network Connectivity in Epilepsy: Resting State fMRI and EEG-fMRI Contributions. Front Neurol 5: 93.

34. Doron I, Haluta E, Baruchi I, Towle VL, Ben-Jacob E (2006) Timeinvariant patient specific frequency templates in human brain. Physical Review Letters.

35. Lachaux JP, Rodriguez E, Martinerie J, Varela FJ (1999) Measuring phase synchrony in brain signals. Hum Brain Mapp 8: 194-208.

36. Burns SP, Santanielloa S, Yaffea RB, Jouny CC, Cronec NE, et al. (2014) Network dynamics of the brain and influence of the epileptic seizure onset zone. PANS 111: 5321-5330.

37. Thompson SA, Alexopoulos A, Bingaman W, Gonzalez-Martinez J, Bulacio J, et al. (2015) Auditory aura in frontal opercular epilepsy: sounds from afar. Epileptic Disord 17: 150-155.

38. Dugan P, Carlson C, Bluvstein J, Chong DJ, Friedman D, et al. (2014) Auras in generalized epilepsy. Neurology 83: 1444-1449.

39. Lohse A, Kjaer TW, Sabers A, Wolf P (2015) Epileptic aura and perception of self-control. Epilepsy Behav 45: 191-194.

40. Velasco F, Velasco M, Velasco AL, Menez D, Rocha L (2002) Electrical stimulation for epilepsy: stimulation of hippocampal foci. Stereotact Funct Neurosurg 77: 223-227.

41. Fisher R, Salanova V, Witt T, Worth R, Henry T, et al. (2010) Electrical stimulation of the anterior nucleus of thalamus for treatment of refractory epilepsy. Epilepsia 51: 899-908.

42. Philippe K, Antoine D (2010) Deep brain stimulation in epilepsy: what is next?. Curren Opini Neurol 23: 177-182. 